\title{
ESTADO DE CONOCIMIENTO DE LOS PLECOPTEROS DE CHILE
}

\section{CURRENT STATE OF KNOWLEDGE OF PLECOPTERA OF CHILE}

\author{
Alejandro Vera \& Ariel Camousseight \\ Museo Nacional de Historia Natural, Casilla 787, Santiago, Chile. \\ E-mail: alveras2@gmail.com.
}

\begin{abstract}
RESUMEN
Se analiza el actual conocimiento del Orden en Chile, enfatizando los aspectos taxonómicos útiles para el trabajo limnológico, como son, el grado de discriminación que es posible lograr a través de los estados preimaginales y la distribución geográfica de las especies. Los resultados obtenidos revelan un total de 63 especies distribuidas en 33 géneros y 6 familias. El endemismo en Chile alcanza un 57\%.

Palabras Claves: Lista taxonómica, Plecoptera, Chile, distribución.
\end{abstract}

\section{ABSTRACT}

The actual knowledge of the Order in Chile was studied, emphasizing in the taxonomic date useful for the limnological work such as the knowledge of the immature state and geographical distribution of species. A total of 63 species distributed in 33 genera and 6 families are recognized, and the endemism reaches $57 \%$ of the species.

Keywords: Taxonomic list, Plecoptera, Chile, distribution.

\section{INTRODUCCION}

El orden Plecoptera es el grupo más primitivo de los llamados "orthopteroideos", se reconocen más de 2000 especies en todo el mundo (Zwick 1973), distribuidos en dos subórdenes y 16 familias (Zwick 2000,2003 ), cuyo origen se relaciona con la disgregación del supercontinente de Pangea. El suborden Antarctoperlaria (Gondwana), con cuatro familias, y el suborden Arctoperlaria (Laurasia), con 12. Algunos elementos del hemisferio norte, se habrían desplazado hacia el sur, como es el caso de Perlidae y posiblemente Notonemuridae, lo que explica su presencia en el cono sur de Sudamérica (Zwick 1973, 2000). Respecto de la ecología, estos organismos presentan ninfas acuáticas que cumplen un importante rol en las cadenas tróficas, encontrándose en diversos sistemas hídricos continentales. En Notonemouridae se han registrado elementos de tipo anfibio (Illies 1961, McLellan 1991) e incluso terrestres de ambientes húmedos, en Gripopterygidae (Illies 1960). En general, para su desarrollo, son muy exigentes de los factores fisicoquímicos, por lo que se les considera excelentes indicadores biológicos de calidad de agua (Theischinger 1991).

El primer Plecóptero descrito para Chile es de larga data, Perla gayi Pictet 1841, que junto a los aportes de Blanchard (1851), Brauer (1869) y Mabille (1891) dejan ocho especies descritas para fines del siglo XIX. Los siguientes estudios, realizados por Navás (1920, $1925,1928,1929,1930,1933$ y 1934), permiten que en el primer catálogo mundial del grupo sean citadas 25 especies para el país (Classen 1940).

Sin duda, los aportes más importantes realizados en el estudio de la fauna nacional han sido los trabajos 
de Aubert (1960) e Illies (1958, 1960a, b, c, d, 1961, 1962, 1963, 1964a, b, c, 1965a, b, 1969). Este último autor, ha contribuido con la descripción de un gran número de especies, además de resolver y organizar el estatus supraespecífico de éstas. Por otra parte, este autor entrega antecedentes biogeográficos y ecológicos, enfatizado por primera vez en el estudio de los estados inmaduros. En un contexto global, gran parte de la información se resume en el catálogo publicado por Illies (1966). El último catálogo mundial de Zwick (1973) reúne algunos nuevos aportes para Chile, además de hacer una propuesta filogenética.

Camousseight (1995) establece y discute la secuencia de eventos taxonómicos relativos al estudio de la fauna nacional, junto con enmendar las omisiones publicadas en el catálogo de plecópteros de Chile (Cekalovic 1976).

Los aportes más recientes se han realizado en el marco de revisiones a nivel de familia para Sudamérica, que permiten organizar aspectos supraespecíficos, entregar nuevas sinonimias y aportar con algunas nuevas especies. Este es el caso de Notonemouridae (McLellan \& Zwick 1996), Perlidae (Stark 2001) y Austroperlidae (McLellan 2001). Debe destacarse que en ningún trabajo se ha modificado el estatus de las cinco especies incertae sedis dadas para Chile.

Respecto de los aportes globales útiles para la determinación de las especies, son las claves de Illies (op. cit.) las más utilizadas, a través de las traducciones de Benedetto (1974). Romero (2001) actualiza estas mismas claves e incorpora los estados ninfales.

En lo que se refiere a aspectos biológicos, no hay estudios específicos para la fauna chilena, salvo las observaciones adicionadas a las descripciones originales, los registros de vuelo Marden \& Thomas (2003) y el análisis de hábitos alimenticios de Albariño (2001).

\section{RIQUEZA TAXONÓMICA}

En la Tabla I se presenta la lista de los Plecópteros de Chile, indicando la distribución y descripciones de adultos y ninfas. A continuación se presenta un breve comentario para cada una de las familias:

a) Eustheniidae: con ninfas depredadoras y presentes sólo en Oceanía y Chile; se encuentra representada por dos géneros monoespecíficos exclusivos del país, los que a su vez pertenecen a subfamilias diferentes, de las tres mundialmente conocidas (Zwick 1997). El buen conocimiento de los estados inmaduros es debido al trabajo de Illies (1960). Se extiende la distribución de Neuroperlopsis patris Illies 1960 a las regiones VII y Metropolitana.

b) Diamphipnoidae: con ninfas detritívoras; es reconocida como exclusiva de Sudamérica. Sólo Diamphipnoa helgae Illies 1960 se encuentra también en Argentina. Diamphipnopsis beschi Illies 1960 se conoce sólo por el Holotipo macho. Las ninfas son conocidas por las descripciones hechas para caracterizar los géneros (Illies 1960).

Respecto de la distribución geográfica existiría un gran desconocimiento en este grupo. Las ninfas de ambos géneros son fácilmente colectadas en variados arroyos de las cordilleras de los Andes y de la Costa, desde la $\mathrm{V}$ a la XI Región.

c) Austroperlidae: con ninfas detritívoras; cuyos representantes se encuentran en Sudamérica y Oceanía. Los géneros presentes en nuestro país están también en Argentina asociados a la Cordillera de los Andes, salvo Klapopteryx armillata Navás 1928. La reciente revisión de McLellan (2001) aporta con la designación del nuevo género Andesobius y deja en sinonimia de Klapopteryx armillata a Klapopteryx costalis, entregando además una clave para la identificación de machos, hembras y ninfas. En relación al conocimiento de los estados inmaduros, a pesar que todas las ninfas son conocidas, el tema no esta agotado puesto que se ha encontrado y no descrito una ninfa no asociable a las especies conocidas (McLellan op. cit.).

d) Gripopterygidae: con ninfas mayoritariamente detritívoras, presente en Oceanía y Sudamérica, es sin duda una de las familias mejor representadas en número y diversidad, distribuida a lo largo de todo el territorio, tanto en sistemas de cordillera como de valles, e incluso en la desembocadura de los ríos en el mar (antecedentes inéditos). La familia ha sido intensamente trabajada, fundamentalmente por Illies (1963), estableciendo un sistema clasificatorio supraespecífico fundado en el conocimiento de los estados inmaduros. Pese a ello, persiste un 55\% de las especies con ninfas desconocidas o bien descritas, pero sólo asignadas a géneros. Es notable el caso de Araucanioperla, del cual se conocen dos especies en base a imagos y tres ninfas diferentes, no asignadas a ninguna de ellas. En esta familia se ubica la única especie compartida con Perú y Bolivia Claudioperla tigrina (Klapalek 1904). 
Plecópteros de Chile: Vera, A. \& A. Camousseight

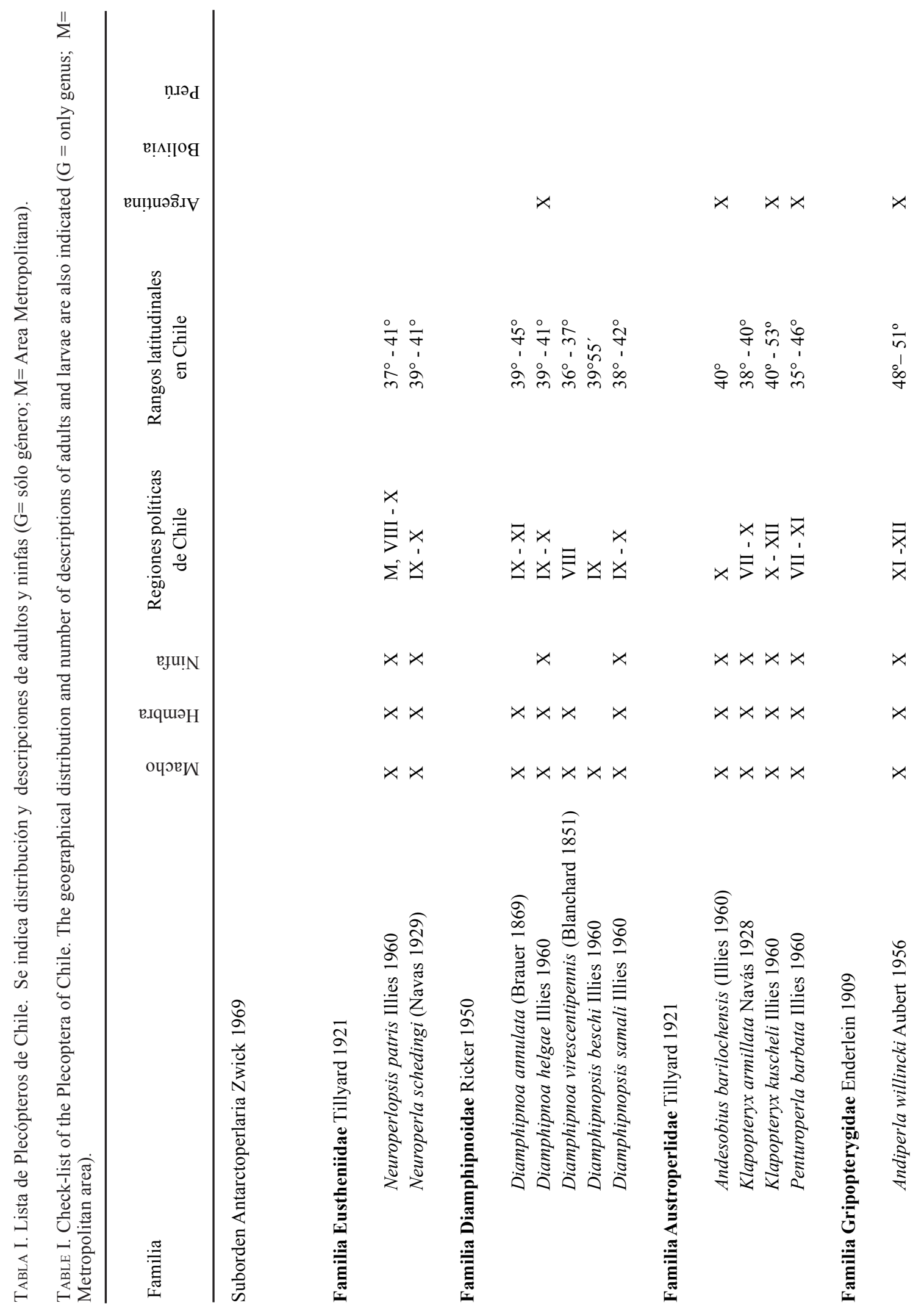


Gayana 70(1), 2006

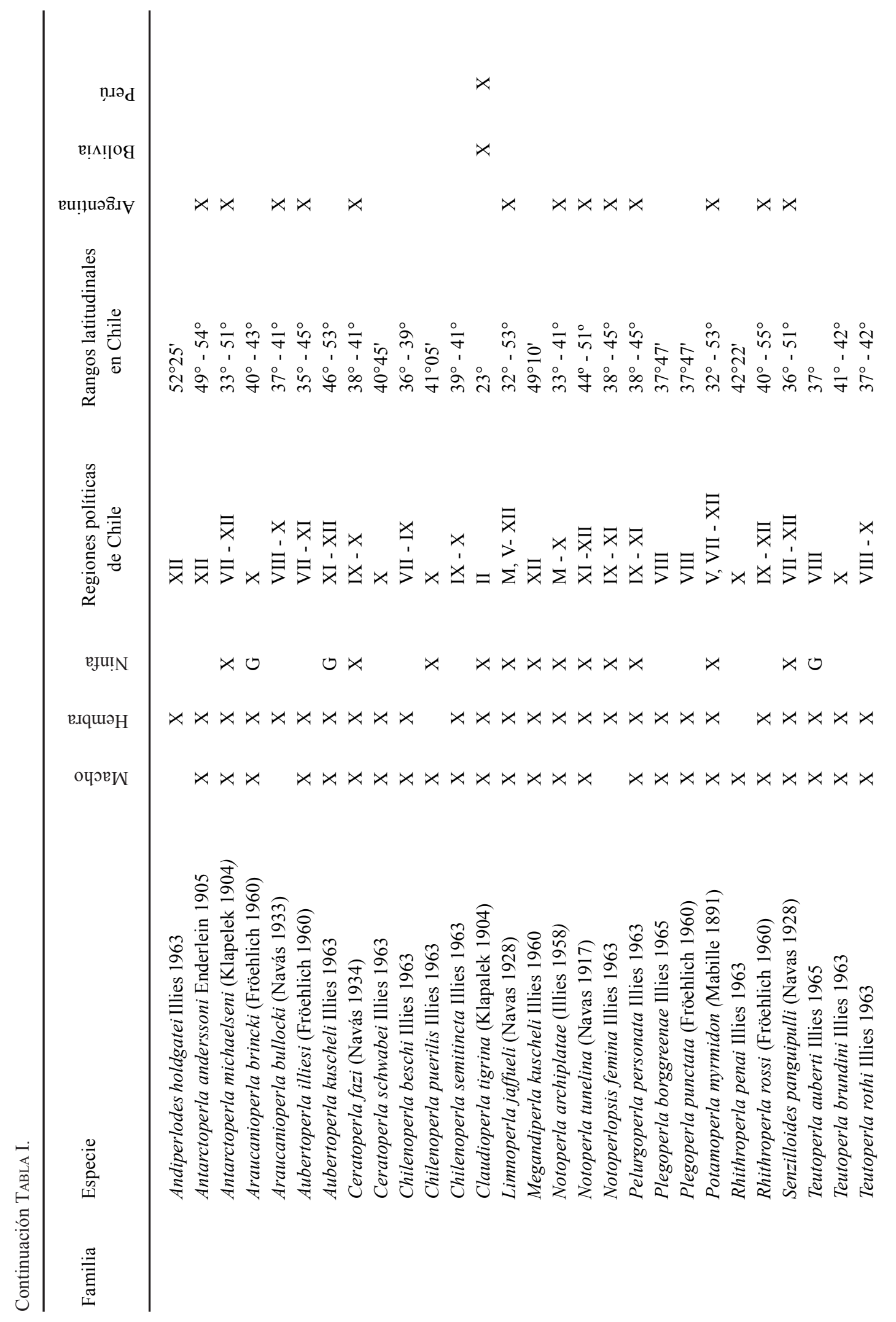


Plecópteros de Chile: Vera, A. \& A. Camousseight

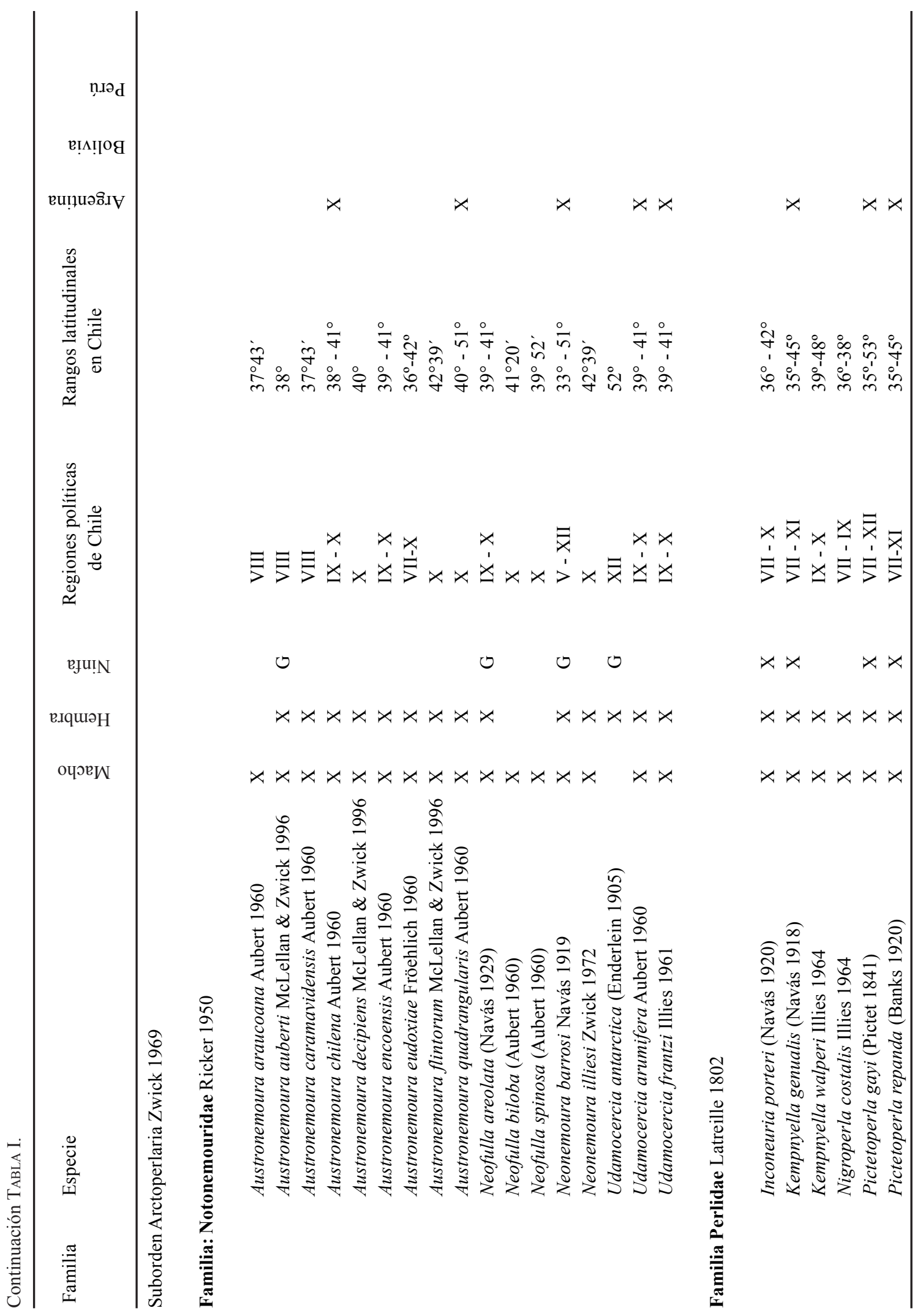


e) Notonemouridae: dieta de ninfas desconocida para las especies chilenas. Forma parte de uno de los grupos más controversiales, constituyendo un conjunto parafilético (Zwick 2000). Se la encuentra en Sudáfrica, India, Oceanía y Sudamérica. El conocimiento taxonómico para el país se ha basado fundamentalmente en el estudio de machos, a los que con posterioridad se les han atribuido hembras, de allí la dudosa situación para muchas de las especies. El conocimiento de los estados inmaduros sólo permite reconocer los géneros (Illies 1961).

f) Perlidae: con ninfas depredadoras, con unas pocas especies en el territorio nacional, pero que sin embargo han logrado un significativo grado de diferenciación genérica. Los cuatro géneros representan el $40 \%$ de los Neotropicales (Stark 2001). Inconeuria posee dos especies, una en el país y la otra es propia de Perú; notable es la brecha entre ambas consecuencia de la ausencia de registros desde el límite norte de Chile con Perú y hasta la VII Región por el sur. Pictetoperla es propia de la Cordillera de los Andes, desde Chillán (VIII Región) al sur. Se extiende hacia el norte la distribución de Kempnyella, con un registro en la localidad de Los Andes V Región. Nigroperla es endémico de la Cordillera de la Costa de Chile, y se amplía el límite norte de su distribución a la VII Región. Respecto del conocimiento de los estados preimaginales, sólo es desconocida la ninfa de Nigroperla. Stark (2001) realizó una sinopsis de los géneros sudamericanos, aportando para la fauna chilena el análisis de los huevos para todos los géneros y la sinonimia de Pictetoperla brundini en Kempnyella genualis, incluyendo además una clave de identificación para machos, hembras y ninfas.

En la Tabla II se presenta un cuadro comparativo sobre el estado de conocimiento de los plecópteros chilenos, señalando el endemismo de cada familia (\%), el número de especies y géneros, y el número de descripciones para machos, hembras y ninfas.

Sin duda la mayor cantidad de especies se presenta en Gripopterygidae, además el mayor número de géneros. Respecto del conocimiento de las especies, las hembras faltantes por describir sólo son casos puntuales, restando aún un importante porcentaje de ninfas desconocidas
$(60,3 \%)$, lo que dificulta los estudios limnológicos. Sin embargo, es posible trabajar con bastante certeza a nivel de géneros, donde el conocimiento alcanza el 91\%.

El endemismo expresado para cada una de las familias responde a los límites políticos del extremo austral de América. Estas cifras enmascaran la real exclusividad de las especies de esta región, ya que estando asociadas a la Cordillera de los Andes, frecuentemente se encuentran tanto en las vertientes orientales como en las occidentales, considerándose de este modo, como elementos presentes también en Argentina. Del total de especies sólo una es compartida con Perú y Bolivia.

\section{RECURSOS HUMANOS Y LOGÍSTICOS}

Estos insectos han sido estudiados fundamentalmente por especialistas extranjeros, de modo que casi la totalidad del material se ha depositado en instituciones foráneas. La única excepción es el Holotipo de Megandiperla kuscheli Illies 1960 depositado en el Museo Nacional de Historia Natural (Camousseight 1980). La formación de colecciones de referencia en el país es un trabajo incipiente, valorándose el material depositado en la facultad de Ciencias Agrarias y Forestales de la Universidad de Chile y la creciente Colección del Museo Nacional de Historia Natural.

En este grupo resta aún una importante fracción de interrogantes, i.e. taxonómicas, de distribución geográfica, aspectos biológicos y ecológicos de las especies. Esta información será de gran utilidad para la determinación de bioindicadores de calidad de agua, además de contribuir al análisis evolutivo del grupo.

El orden presenta gran riqueza de líneas de investigación, algunas de las cuales están siendo retomadas por el primer autor de este trabajo.

\section{ESTADO DE CONSERVACIÓN}

El escaso conocimiento biológico del grupo hace imposible evaluar su actual situación de conservación, pero la gran diversidad y endemismo de especies concentradas entre la IX y X regiones hace pensar en la fragilidad de ellas frente a las modificaciones de sus sistemas hídricos. Hay que señalar además que la inexistencia de registros en gran parte del territorio nacional impide emitir un juicio más acabado. 
Tabla II. Conocimiento del orden en Chile. Se indica el porcentaje de endemismo para cada familia, el número de especies y géneros, las descripciones para machos, hembras y ninfas.

TABLE II. Knowledge of the order in Chile. The number of species and genera for each family, the number of descriptions for male, female and larvae, and the endemism, are indicated.

\begin{tabular}{lccccccc}
\hline Familia & \multicolumn{2}{c}{ Diversidad } & \multicolumn{3}{c}{ Número de descripciones } & \multicolumn{2}{c}{ Endemismo } \\
& Especies & Géneros & Machos & Hembras & Ninfas & Ninfas de géneros & \\
\hline Eustheniidae & 2 & 2 & 2 & 2 & 2 & 2 & 100 \\
Diamphipnoidae & 5 & 2 & 5 & 4 & 2 & 2 & 80 \\
Austroperlidae & 4 & 3 & 4 & 4 & 4 & 3 & 45 \\
Gripopterygidae & 29 & 18 & 26 & 27 & 13 & 4 & 70 \\
Notonemouridae & 17 & 4 & 16 & 13 & 0 & 3 & 50 \\
Perlidae & 6 & 4 & 6 & 6 & 4 & 30 & 57 \\
\hline Total & 63 & 33 & 59 & 56 & 25 & \multirow{2}{*}{5}
\end{tabular}

\section{BIBLIOGRAFIA}

Albariño, R. J. 2001. The food habits and mounthpart morphology of a South Andes population of Klapopteryx kuscheli (Plecoptera: Austroperlidae). Aquatic Insects 23(3): 171-181.

Aubert, J. 1956. Andiperla willincki n. sp., Plécoptère nouveau des Andes de Patagonie. Mitteilungen der Schweizerischen Entomologischen Gesellschaft 29(2): 229-232.

Aubert, J. 1960. Contribution a l'étude des Notonemourinae (Plecoptera) de l'Amérique du Sud. Mitteilungen der Schweizerischen Entomologischen Gesellschaft 33(1-2): 47-64.

Benedetto, L. 1974. Clave para la determinación de los Plecópteros sudamericanos. Studies Neotropical Fauna and Environment 9: 141 - 170.

Blanchard, E. 1951. Perlinos. En: Historia Física y Política de Chile 6 (Ed. C. Gay), pp. 96-102. Maulde \& Renou, Paris.

Brauer, F. 1869. Stenoperla annulata n.sp. Chile. Beschreibung neuer Neuropteren aus dem Museum Godeffroy in Hamburg. Verhandlungen der Kaiserlinch-königlichen zoologischbotanischen Gesellschaft in Wien 19:17-18.

Camousseight, A. 1980. Catálogo de tipos de insecta depositados en la colección del Museo Nacional de Historia Natural (Santiago, Chile). Publicación Ocasional Museo Nacional de Historia Natural Chile 32:1-45.

Camousseight, A. 1995. Plecoptera. En: Diversidad Biológica de Chile (Eds. J.A. Simonetti, M.T.K. Arroyo, A.E. Spotorno y E. Lozada), pp. 218-223. Comisión Nacional de Investigación Científica y Tecnológica, Santiago de Chile.

Cekalovic, T. 1976. Catálogo de los Plecópteros de Chile. Boletín de la Sociedad de Biología de Concepción 50: 145-156.
Classen, P.W. 1940. A catalogue on the Plecoptera of the world. Memoir Cornell University Agriculture Experiment Station 232: 1-235.

Fröehlich, C.G. 1960. Some Gripopterygids and Notonemourines (Plecoptera) from South America. Lunds Universitets Arsskrift 56(13):123.

Illies, J. 1958. Abranchioperlidae, eine neue Plecopterenfamilie. Investigaciones Zoológicas Chilenas 4 :223-230.

ILLIES, J. 1960a. Archiperlaria, eine neue Unterordung der Plecopteren (Revision der Familien Eustheniiden und Diamphipnoiden). Beitrage zur Entomologie 10 (7-8): 661-697.

Illies, J. 1960b. Penturoperlidae, eine neue Plecopterenfamilie. Zoologischer Anzeiger (Leipzig) 164: 26-41.

Illies, J. 1960c. Die erste auch im Larvenstadium terrestrische Plecoptere. Mitteilungen der Schweizerischen Entomologischen Gesellschaft 33(3): 161-168.

ILLIES, J. 1960d. Zur Kenntnis der neuen Plecopterenfamilie Abranchioperlidae. Archiv für Hydrobiologie 56 (4): 413-424.

ILLIES, J. 1961. Südamerikanische Notonemouinae und die Stellung der Unterfamilie im System der Plecopteren. Mitteilungen der Schweitzerischen Entomologischen Gesellschaft 34 (2): 97-126.

Illies, J. 1962. Die Unterordungen, Familien und Gattungen der Plecoptera. Verhandlungen Intern. Konger. Entomol. 11 ${ }^{\text {th }}$ Wien (3): 263-267.

Illies, J. 1963. Revision des südamerikanischen Grypopterygidae (Plecoptera). Mitteilungen der Schweitzerischen Entomologischen Gesellschaft 36 (3): 145-248.

IlliES, J. 1964a. Südamerikanische Perlidae (Plecoptera) besonders aus Chile und Argentinien. Beiträge zur Neotropischen Fauna 3(3): 207-233. 
Gayana 70(1), 2006

Illies, J. 1964b. Neue Plecopteren aus Südamerika. Gewässer und abwässer 36:49-57.

Illies, J. 1964c. Die plecopteren-Unterfamilie Anchiperlinae (Auflösung einer unnatürlichen Negativgruppe nach Remane). Zoologischer Anzeiger (Leipzig) 172 (1): 37-48.

Illies, J. 1965a. Neue Plecopteren aus Chile und Argentinien. Mitteilungen der Schweizerischen Entomologischen Gesellschaft 37 (3): 151-156.

Illies, J. 1965b. Phylogeny and zoogeography of the Plecoptera. Annual Review of Entomology 10: 117-140.

Illies, J. 1969. Revision der Plecopteren familie Austroperlidae. Entomologisk Tidskrift 90(1-2): 19-51.

ILLIES, J. 1977. Plecoptera. En: Biota Acuática de Sudamérica Austral (Ed. S.A. Hubert), pp. 185-186. San Diego State University, San Diego.

Lanfranco, D. 1982. Presencia de Archiperla willinki en Chile. Anales del Instituto de la Patagonia 13: 207209.

Mabille, P. 1891. Néuroptères. Zoologie Missions Scientifique du Cap Horn 1882-1883 6(2): 6-9.

Marden, J. H., B. C. O’Donnell, M. A. Thomas \& J. Y. Bye. 2000. Surface-skimming stoneflies and mayflies: The taxonomic and mechanical diversity of two-dimensional aerodynamic locomotion. Physiological and Biochemical Zoology 73(6):751764.

Marden, J. H. \& M. A. Thomas. 2003. Rowing locomotion by a stonefly that possesses the ancestral pterygote condition of co-occurring wings and abdominal gills. Biological Journal of the Linnean Society 79(2): 341-349.

McLellan, I.D. 1991. Notonemouridae (Insecta: Plecoptera). Fauna New Zealand 22: 1-64.

McLellan, I.D. \& P. Zwick. 1996. Austronemoura auberti new species and other new Chilean Notonemouridae (Plecoptera). Mitteilungen der Schweizerischen Entomologischen Gesellschaft 69: 107-115.

McLellan, I.D. 2001. A Revision of South American Austroperlidae (Plecoptera). Aquatic Insects 23(3):233-251.

Navas, L. 1920. Insecta nova. Memorie della Pontificia Accademia Romana dei Nuovi Lincei Serie 2, 5: 2-7.
NAVAS, L. 1925. Insectos Neotrópicos. $1^{\text {a }}$ Serie. Revista Chilena de Historia Natural 29:305-313.

NAVAS, L. 1928. Insectos Neotrópicos. $4^{\mathrm{a}}$ Serie. Revista Chilena de Historia Natural 32:106-128.

Navas, L. 1929. Algunos insectos de Chile. $3^{\text {a }}$ Serie. Revista Chilena de Historia Natural 33: 326-334.

Navas, L. 1930. Algunos insectos de Chile. $4^{\text {a }}$ Serie. Revista Chilena de Historia Natural 34: 350-366.

Navas, L. 1933. Algunos insectos de Chile. $4^{\text {a }}$ Serie. Revista Chilena de Historia Natural 37: 230-234.

NAVAS, L. 1934. Insectos sudamericanos. $9^{a}$ Serie. Revista de la Real Academia de Ciencias Exactas, Físicas y Naturales de Madrid 31 (11): 155-184

Pictet, F.J. 1841. Historie naturalle générale et particulière des onsects Néuroptéres Famille des Perlides: Kess. Man, Genève 1-423.

Romero, V.F. 2001. Plecoptera. En: Guía para la determinación de los artrópodos bentónicos sudamericanos (Eds. H. Fernández y E. Domínguez), pp. 93-109. Facultad de Ciencias Naturales e Instituto Miguel Lillo, Tucumán, Argentina.

STARK, B.P. 2001. A synopsis of neotropical Perlidae (Plecoptera). En: Trends in research in Ephemeroptera and Plecoptera (Ed. E. Domínguez), pp. 405-422. Springer.

Theischinger, G. 1991. Plecoptera. En: The insects of Australia (Ed. G. Theischinger), pp. 311-319. Carlton, Melbourne University Press.

Zwick, P. 1972. On Neonemura illiesi nov. spec. and some other stoneflies from Chile (Plecoptera). Studies on the Neotropical Fauna and Environment 7: 95-100.

Zwick, P. 1973. Insecta: Plecoptera Phylogenetisches. System und Katalog Das Tierreich 94: 465 pp.

Z WICK, P. 1979. Revision of the stonefly family Eustheniidae (Plecoptera), with emphasis on the Fauna of the Australian Region. Aquatic Insects 1(1): 17-50.

ZwICk, P. 2000. Phylogenetic system and zoogeography of the Plecoptera. Annual Review of Entomology 45: 709-746.

ZwICK, P. 2003. Morfological support of the major clades of Plecoptera. Entolmologische Abhandlungen 61(2): 128-130.

Fecha de recepción: 16.08 .05

Fecha de aceptación: 05.12.05 